\title{
IS THERE A METALLICITY CEILING TO FORM CARBON STARS?-A NOVEL TECHNIQUE REVEALS A SCARCITY OF C STARS IN THE INNER M31 DISK*
}

\author{
M. L. Boyer ${ }^{1,2}$, L. Girardi ${ }^{3}$, P. Marigo ${ }^{4}$, B. F. Williams ${ }^{5}$, B. Aringer ${ }^{6}$, W. Nowotny ${ }^{6}$, P. Rosenfield ${ }^{5}$, C. E. Dorman ${ }^{7}$, \\ P. Guhathakurta ${ }^{7}$, J. J. Dalcanton ${ }^{5}$, J. L. Melbourne ${ }^{8}$, K. A. G. Olsen ${ }^{9}$, and D. R. Weisz ${ }^{5}$ \\ ${ }^{1}$ Observational Cosmology Lab, Code 665, NASA Goddard Space Flight Center, Greenbelt, MD 20771, USA; martha.boyer@ nasa.gov \\ ${ }^{2}$ Oak Ridge Associated Universities (ORAU), Oak Ridge, TN 37831, USA \\ ${ }^{3}$ Osservatorio Astronomico di Padova-INAF, Vicolo dell'Osservatorio 5, I-35122 Padova, Italy \\ ${ }^{4}$ Department of Physics and Astronomy G. Galilei, University of Padova, Vicolo dell'Osservatorio 3, I-35122 Padova, Italy \\ ${ }^{5}$ Department of Astronomy, University of Washington, Box 351580, Seattle, WA 98195, USA \\ ${ }^{6}$ Department of Astrophysics, University of Vienna, Türkenschanzstraße 17, A-1180 Wien, Austria \\ ${ }^{7}$ University of California Observatories/Lick Observatory, University of California, 1156 High Street, Santa Cruz, CA 95064, USA \\ ${ }^{8}$ Caltech Optical Observatories, Division of Physics, Mathematics and Astronomy, Mail Stop 301-17, California Institute of Technology, Pasadena, CA 91125, USA \\ ${ }^{9}$ National Optical Astronomy Observatory, 950 North Cherry Avenue, Tucson, AZ 85719, USA \\ Received 2013 May 23; accepted 2013 July 12; published 2013 August 19
}

\begin{abstract}
We use medium-band near-infrared (NIR) Hubble Space Telescope WFC3 photometry with model NIR spectra of asymptotic giant branch (AGB) stars to develop a new tool for efficiently distinguishing carbon-rich (C-type) AGB stars from oxygen-rich (M-type) AGB stars in galaxies at the edge of and outside the Local Group. We present the results of a test of this method on a region of the inner disk of M31, where we find a surprising lack of $\mathrm{C}$ stars, contrary to the findings of previous $\mathrm{C}$ star searches in other regions of M31. We find only one candidate $\mathrm{C}$ star (plus up to six additional, less certain $\mathrm{C}$ star candidates), resulting in an extremely low ratio of $\mathrm{C}$ to $\mathrm{M}$ stars $\left(\mathrm{C} / \mathrm{M}=\left(3.3_{-0.1}^{+20}\right) \times 10^{-4}\right)$ that is one to two orders of magnitude lower than other $\mathrm{C} / \mathrm{M}$ estimates in $\mathrm{M} 31$. The low $\mathrm{C} / \mathrm{M}$ ratio is likely due to the high metallicity in this region which impedes stars from achieving $\mathrm{C} / \mathrm{O}>1$ in their atmospheres. These observations provide stringent constraints to evolutionary models of metal-rich AGB stars and suggest that there is a metallicity threshold above which $\mathrm{M}$ stars are unable to make the transition to $\mathrm{C}$ stars, dramatically affecting AGB mass loss and dust production and, consequently, the observed global properties of metal-rich galaxies.
\end{abstract}

Key words: galaxies: individual (M31) - stars: AGB and post-AGB - stars: carbon - stars: late-type

Online-only material: color figures

\section{INTRODUCTION}

Asymptotic giant branch (AGB) stars play a significant role in galaxies' observed properties and their evolution. They are responsible for a major share of galaxy luminosity (e.g., Maraston et al. 2006; Marigo et al. 2010; Boyer et al. 2011; Melbourne et al. 2012; Melbourne \& Boyer 2013) and contribute considerably to the chemical enrichment of the interstellar medium (e.g., Marigo 2001; Ventura et al. 2001; Karakas \& Lattanzio 2007). Despite its widespread importance, the AGB phase remains among the most uncertain phases of stellar evolution modeling, leading to the largest uncertainties in galaxy stellar population synthesis (Conroy et al. 2009).

AGB atmospheres have complicated elemental abundances due primarily to the third dredge-up (3DU) process, wherein they pull newly synthesized carbon and other elements to the surface. The carbon bonds with free oxygen to make $\mathrm{CO}$, and the excess oxygen or carbon dictates the molecular and dust chemistry of the star (Marigo \& Aringer 2009; Ferrarotti \& Gail 2006), having drastic consequences for the mass-loss and dust-production processes. The ratio $(\mathrm{C} / \mathrm{M})$ of $\mathrm{C}$ stars (those AGB stars with excess free carbon in their atmospheres) to $\mathrm{M}$ stars (those with excess free oxygen) generally decreases with increasing metallicity (e.g., Rowe et al. 2005; Groenewegen 2006a, 2007; Battinelli \& Demers 2005). Metal-poor stars are

\footnotetext{
* Based on observations made with the NASA/ESA Hubble Space Telescope, obtained from the Data Archive at the STScI, which is operated by the AURA, Inc., under NASA contract NAS5-26555.
}

more likely to become $\mathrm{C}$-rich because less oxygen is available to bind newly dredged-up carbon into $\mathrm{CO}$ and because the depth of 3DU events increases at low metallicity (Karakas et al. 2002). Both factors favor the formation of carbon stars after fewer and fewer dredge-up events at decreasing metallicity. Although this effect is well established on theoretical grounds, quantitative predictions of the $\mathrm{C} / \mathrm{M}$ ratio from first principles are not straightforward, given the critical dependence of $\mathrm{C} / \mathrm{M}$ on the star formation history of the parent galaxy and the uncertain details of 3DU modeling. Empirically, the relationship between the $\mathrm{C} / \mathrm{M}$ ratio and metallicity is poorly constrained owing to small number statistics and other sample biases, especially at high metallicity (Figure 1).

\subsection{Carbon Star Surveys}

Carbon star surveys in nearby dwarf galaxies have helped inform evolutionary models at low metallicity (e.g., Battinelli et al. 2007; Battinelli \& Demers 2009; Groenewegen 2006a, 2007, and references therein; Figure 1). However, the majority of these surveys were conducted entirely at optical wavelengths $(<9000 \AA)$ where dusty AGB stars are often undetected owing to circumstellar extinction (e.g., Nowotny et al. 2013). In the Magellanic Clouds, $\approx 30 \%$ of $\mathrm{C}$ stars are fainter than $M_{\mathrm{I}}=$ $-2.5 \mathrm{mag}$ (Boyer et al. 2011), which is the limiting magnitude of the surveys mentioned above. More complete AGB samples can be created using observations in the near-infrared (NIR) where circumstellar dust extinction is less severe. Several studies have searched for carbon stars using NIR photometry in metal-poor 


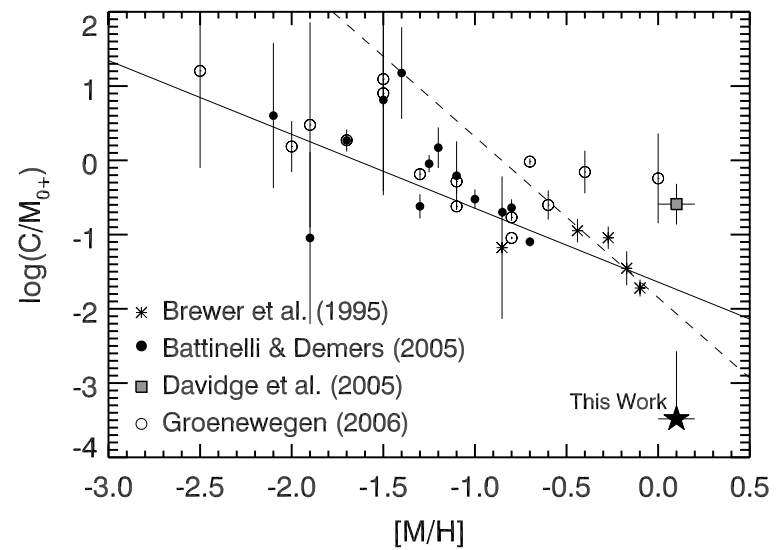

Figure 1. The C/M ratio, adapted from Groenewegen (2006a) and Battinelli \& Demers (2005) for nearby galaxies. Data for five regions in M31 from Brewer et al. (1995) are plotted with asterisks, with metallicities from Zurita \& Bresolin (2012). These data were derived using optical photometry, so the number of $\mathrm{C}$ stars is underestimated by $\approx 20 \%-60 \%$ (e.g., Boyer et al. 2009). The error bars are derived solely from Poisson statistics and do not include information on detection biases or photometric uncertainties. The solid line is fit through all points, and the dashed line is fit only through the Brewer et al. (1995) points. The Davidge et al. (2005) point (gray square) and the point from this work (star) are derived from NIR photometry, with the metallicity range from Saglia et al. (2010). We note that the metallicities for M31 are not measured directly for the fields included here, but are instead derived from estimates of the metallicity gradient across the disk (Zurita \& Bresolin 2012) or the bulge (Saglia et al. 2010).

dwarf galaxies (e.g., Cioni et al. 2006; Sibbons et al. 2012). At higher metallicity, NIR searches are much more difficult owing either to the distance of the galaxy (in the case of M31) or to sample biases and uncertain distance measurements (in the case of the Milky Way).

There are two examples of photometrically complete NIR surveys of AGB stars in the inner region of M31: Stephens et al. (2003), who used the Hubble Space Telescope (HST) NICMOS camera, and Davidge et al. (2005), who used ground-based adaptive optics. Both of these surveys find examples of stars with very red $J-K$ colors. Stephens et al. (2003) identify these stars as long period variables, but do not comment on whether they are $\mathrm{C}$ or $\mathrm{M}$ stars. Davidge et al. (2005) identify them as candidate $\mathrm{C}$ stars based on their NIR colors, suggesting the presence of a large $C$ star population. This is in contrast to $C$ star surveys in the Milky Way bulge, which show an almost total lack of intrinsic C stars (e.g., Azzopardi et al. 1991; Whitelock 1993; Feast 2007; Miszalski et al. 2013). Whether or not these red stars in the M31 bulge and inner disk are C stars remains unexplored, since narrow-band photometry and spectroscopy are difficult both due to limited sensitivity and crowding.

The WFC3 NIR imaging camera on board HST has allowed for high-resolution, sensitive imaging up to $1.6 \mu \mathrm{m}$, enabling observations of AGB stars in galaxies farther than $\sim 5 \mathrm{Mpc}$ for testing and improving models of AGB mass loss (e.g., Melbourne et al. 2012). However, it is impossible to distinguish C- and M-type AGB stars with the available WFC3 broad-band filters alone (Dalcanton et al. 2012a, 2012b). The medium-width filters available for WFC3/IR, on the other hand, do provide the opportunity to separate AGB subtypes (Section 2.1). NIR synthetic spectra of AGB stars (Aringer et al. 2009; B. Aringer et al., in preparation) suggest that these filters can sample individual molecular features in C-rich AGB stars. Therefore, these medium-band filters should provide an efficient way of discriminating between $\mathrm{C}$ - and O-rich AGB stars.
To test this approach, we targeted a field from Davidge et al. (2005) with the WFC3/IR medium-band filters so that any candidate $\mathrm{C}$ stars detected could be compared to those identified via their JHK colors. The Davidge et al. (2005) results suggest that a WFC3/IR single field should encompass a population of several hundred carbon stars, based on the fraction of AGB stars with red $J-K$ and $H-K$ colors in their small $22^{\prime \prime}$ field. However, our observations reveal the near-absence of $C$ stars.

We have organized this paper as follows. In Section 2, we detail our observations and analysis. Section 3 describes our early results regarding the presence of C- and M-type AGB stars. Finally, the paper is summarized and the implications discussed in Section 4.

\section{THE DATA}

Data were collected in a Cycle 20 HST program (GO-12862, PI: Boyer) and consist of $600 \mathrm{~s}$ total integrations $(300 \mathrm{~s} \times$ 2 frames) in each one of the medium-band WFC3/IR filters: F098M, F127M, F139M, and F153M. The 2'.3 × 2'.1 field of view is centered at $\alpha=00^{\mathrm{h}} 43^{\mathrm{m}} 21^{\mathrm{s}} .7, \delta=+41^{\circ} 21^{\prime} 46^{\prime \prime} .2$, approximately $2 \mathrm{kpc}$ from the center of M31 (Figure 2). Photometry was produced using the software package DOLPHOT 2.0 (Dolphin 2000), which uses TINYTIM point-spread functions (PSFs; Krist 1995; Hook et al. 2008) to measure stellar fluxes simultaneously in each image. Unreliable measurements were culled from the DOLPHOT output using the crowding $\left(\left\langle C_{\lambda}\right\rangle>\right.$ $0.25)$, sharpness $\left(\Sigma\left|S_{\lambda}\right|>1\right)$, and signal-to-noise $(\mathrm{S} / \mathrm{N}<3)$ parameters, resulting in a total of 42,940 sources detected in all four filters.

We performed false-star tests to determine the photometric completeness and uncertainty, wherein we added $\approx 50,000$ stars (one at a time) using the appropriate PSFs into each image and reran the photometry as described above. The fake stars were randomly distributed spatially and mimic the observed color-magnitude distribution. The resulting 50\% completeness, or limiting magnitude, is $21.6<m_{50 \%}<22.8 \mathrm{mag}$, with the bluer filters showing better completeness.

To these data, we match the stellar photometric catalogs ${ }^{10}$ in the WFC3/IR F110W and F160W filters from the Panchromatic Hubble Andromeda Treasury (PHAT) program (Dalcanton et al. 2012a). We made no correction for interstellar extinction because it is expected to be low in these filters and will have minimal effect on the separation of C and M stars in F127M - F139M versus F139M - F153M (Section 2.1). Barmby et al. (2000) find $E(B-V)$ as high as $0.4 \mathrm{mag}$ (or $E\left(J-K_{\mathrm{s}}\right) \lesssim 0.1 \mathrm{mag}$; Rieke $\&$ Lebofsky 1985) for globular clusters near the observed field, which can cause a shift of $<0.02 \mathrm{mag}$ in these colors. Figure 3 shows the color-magnitude diagrams (CMDs) and includes the $\mathrm{C}$ star candidates from Davidge et al. (2005) with $H-K \gtrsim 0.4$ (the photometry was redone by Olsen et al. 2006) and candidate $\mathrm{C}$ stars identified by the medium-band WFC3/IR colors (Section 2.1). The thin red giant branch indicates minimal differential extinction across the field.

\subsection{Identifying Carbon Star Candidates}

The location of the change in slope in the luminosity function indicates that the tip of the red giant branch (TRGB) is $M_{\mathrm{F} 153 \mathrm{M}}^{\mathrm{TRGB}}=$ $18.45 \pm 0.10 \mathrm{mag}$, or $M_{\mathrm{F} 153 \mathrm{M}}=-6.0 \mathrm{mag}$ for a distance modulus of $24.45 \mathrm{mag}$ (Dalcanton et al. 2012a). The vast majority of thermally pulsing (TP-)AGB stars should lie above

\footnotetext{
10 http://archive.stsci.edu/prepds/phat/
} 


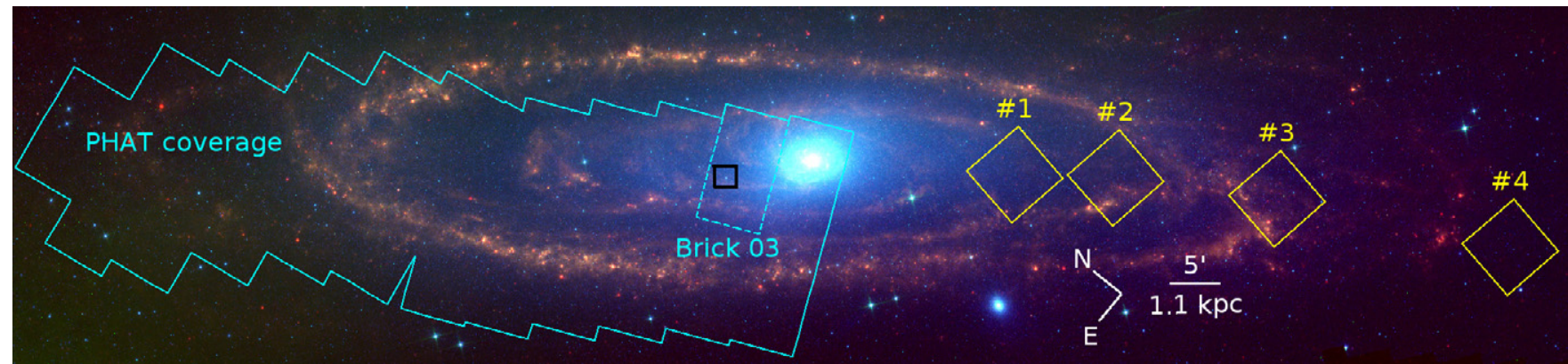

Figure 2. Three-color image of M31 from Spitzer (Barmby et al. 2006). $24 \mu \mathrm{m}$ is plotted in red, $5.8 \mu \mathrm{m}$ in green, and $3.6 \mu \mathrm{m}$ in blue. The PHAT coverage is shown in cyan (Dalcanton et al. 2012a). The coverage discussed here is shown in black and is part of the PHAT survey's "Brick 03." Four of the regions from Brewer et al. (1995) plotted in Figure 1 are shown in yellow. Region \#5 is off the image to the southwest.

(A color version of this figure is available in the online journal.)

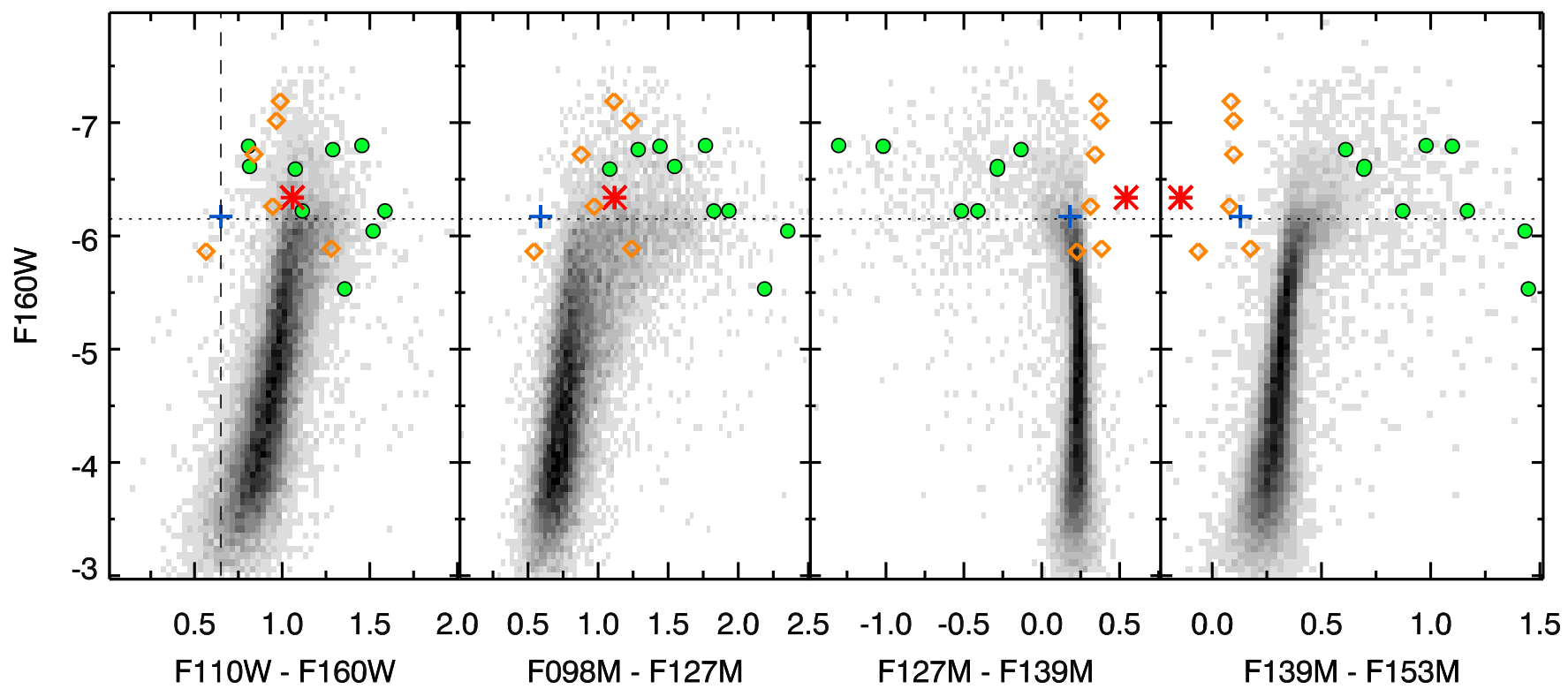

Figure 3. Color-magnitude diagrams, plotted as Hess diagrams to show the point-source density. F160W and F110W magnitudes come from the PHAT survey (Dalcanton et al. 2012a). Sources with $H-K>0.4 \mathrm{mag}$ from Davidge et al. (2005) are plotted as large green circles. The horizontal dotted line is the F160W TRGB from Dalcanton et al. (2012a). The one carbon star candidate identified in Figure 5 is shown as a red asterisk, and the six sources that fall on the edge of the region covered by the carbon star models in Figure 5 are plotted as orange diamonds. An additional star on the edge of M star model coverage in Figure 5 is plotted as a blue plus symbol. This star has colors consistent with a foreground M dwarf (dashed line; also see Figure 21 from Dalcanton et al. 2012a), as does one of the C star candidates.

(A color version of this figure is available in the online journal.)

this limit, with exceptions for a subset of stars experiencing minimum flux during a thermal pulse cycle and very heavily enshrouded stars. We find 3032 AGB stars that are brighter than the TRGB; those that are not classified as $\mathrm{C}$ stars are considered to be $\mathrm{M}$ stars.

To identify C stars, we employ the color combination of F127M - F139M versus F139M - F153M, which is insensitive to circumstellar and interstellar extinction and takes advantage of molecular features present in AGB spectra. For example, the F153M filter falls entirely within a broad absorption feature of $\mathrm{C}_{2}+\mathrm{CN}$ (Figure 4), with its bandhead at $1.4 \mu \mathrm{m}$, which is common in C-rich stars. O-rich AGB stars of early M subtypes have no significant absorption feature in the range from $\sim 1$ to $1.6 \mu \mathrm{m}$, whereas those of late $\mathrm{M}$ subtypes develop a wide $\mathrm{H}_{2} \mathrm{O}$ absorption feature between 1.3 and $1.55 \mu \mathrm{m}$ which can be sampled with the F139M filter. Figure 5(B) shows the location of model atmospheres for objects of $\mathrm{M}$ and $\mathrm{C}$ type from Aringer et al. (2009) and B. Aringer et al. (in preparation) in color space, as compared to our WFC3 data. Surprisingly, only one star re- sides firmly in the region where $\mathrm{C}$ stars are expected, and only six additional sources fall on the edge of the color-color space covered by the $\mathrm{C}$ star models (Figure 5(A)), far fewer than what is predicted by the relationship shown in Figure 1 (Section 3). It is unclear whether or not the six stars near the border drawn in Figure 5(B) are true carbon stars. However, we verify via visual inspection that they are real point-sources and are unaffected by imaging artifacts and/or crowding. To push any of these marginal candidates outside of the shaded region via correction for interstellar extinction, $E\left(J-K_{\mathrm{s}}\right)>0.8 \mathrm{mag}$ is required. This is higher than the expected extinction (Section 2); however, we note that the shaded region in Figure 5 is approximate.

To the left of the shaded region in Figure 5 and below the bulk of the $\mathrm{M}$ star population are an additional 8 sources $(0.06<$ F127M - F139M $<0.18$ mag and $0.13<$ F139M - F153M $<$ 0.23 ). Models from B. Aringer et al. (in preparation) indicate that these stars may be M dwarfs. TRILEGAL simulations in Dalcanton et al. (2012a) predict that a single WFC3/IR field should contain $\approx 6$ foreground $\mathrm{M}$ dwarf stars. Of these eight 

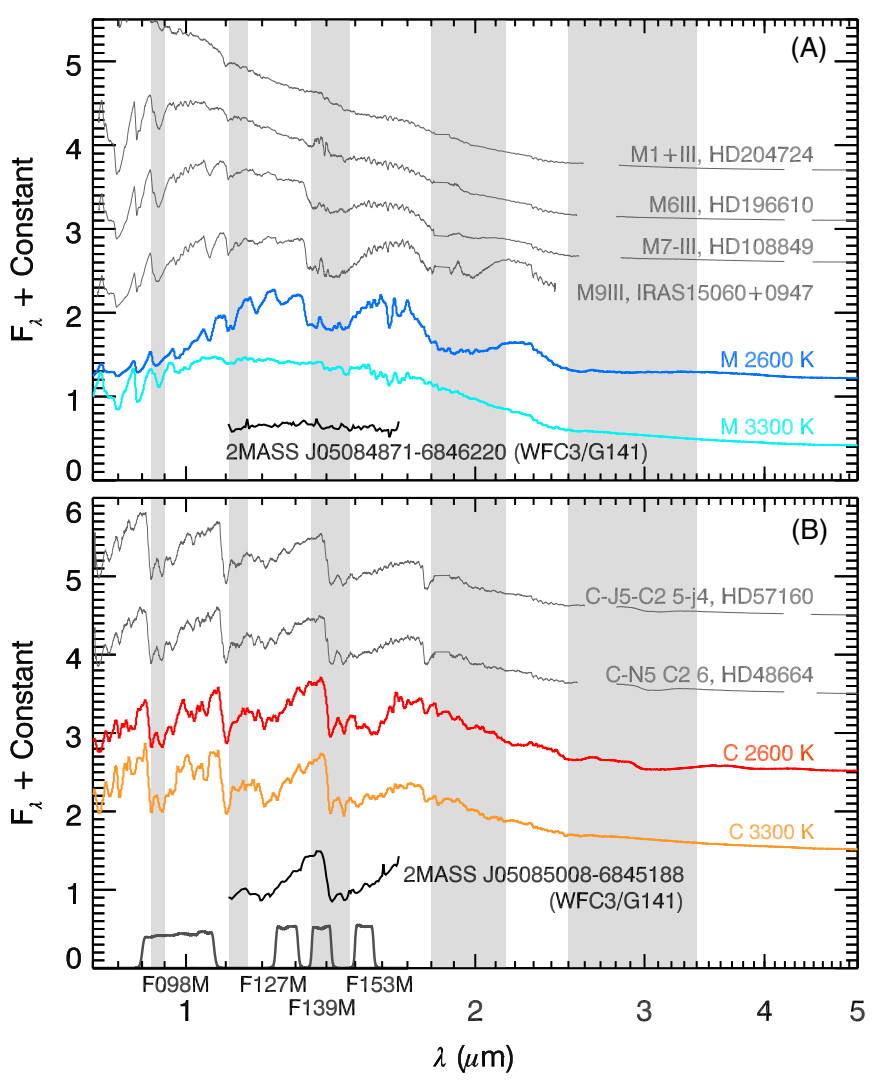

Figure 4. IRTF spectra of Galactic stars (Rayner et al. 2009), plotted in gray, for (A) M stars in order of increasing water absorption and (B) C stars. Solar metallicity model spectra from Aringer et al. (2009) and B. Aringer et al. (in preparation) are also plotted for (A) M stars (blue/cyan) and (B) C stars (red/orange) with $T_{\text {eff }}=2600 \mathrm{~K}$ and $3300 \mathrm{~K}$. Shaded regions mark wavelengths affected by telluric absorption. The model spectra predict all major features present in the IRTF spectra. Also shown in black are telluric-free spectra of two stars in NGC 1850 across the spectral region covered by the WFC3/IR G141 grism: panel (A) shows the M star 2MASS J05084871-6846220 and panel (B) shows the C star 2MASS J05085008-6845188. These two stars present 2MASS colors and magnitudes typical of most C-rich and O-rich AGB stars in the LMC and do not show any surprising features in the F139M band.

(A color version of this figure is available in the online journal.)

stars, five are covered by the currently available PHAT catalog, and three of these (including the one closest to the $\mathrm{C}$ star region, marked by a blue plus in Figure 5(A)) have F110W - F160W colors consistent with $\mathrm{M}$ dwarf stars (Figure 3; also see Figure 21 from Dalcanton et al. 2012a). Correction for any possible interstellar or circumstellar extinction cannot move these stars into the $\mathrm{C}$ star region. We thus conclude that none of these eight stars are candidate $\mathrm{C}$ stars. We also note that one of the marginal candidate $\mathrm{C}$ stars within the shaded region of Figure 5 shows $\mathrm{F} 110 \mathrm{~W}-\mathrm{F} 160 \mathrm{~W}=0.57 \mathrm{mag}$, a color that is consistent with M dwarf stars.

Very dusty carbon stars can be undetected even at NIR wavelengths. Reddening vectors in Figure 5 show that our method is very insensitive to circumstellar extinction. A dustenshrouded star with $E\left(J-K_{\mathrm{s}}\right)=1 \mathrm{mag}$-for which any colorbased $\mathrm{C} / \mathrm{M}$ classification using ground-based $J H K$ photometry would be unreliable - is marginally affected in the WFC3/IR medium-band filters, with color excesses $E(\mathrm{~F} 127 \mathrm{M}-\mathrm{F} 139 \mathrm{M})$ and $E(\mathrm{~F} 139 \mathrm{M}-\mathrm{F} 153 \mathrm{M})$ smaller than $0.2 \mathrm{mag}$. This has an effect only at the edge of the $\mathrm{C}$ star region, with the possibility that the six stars near the $\mathrm{C}$ star border are instead very dusty M stars.

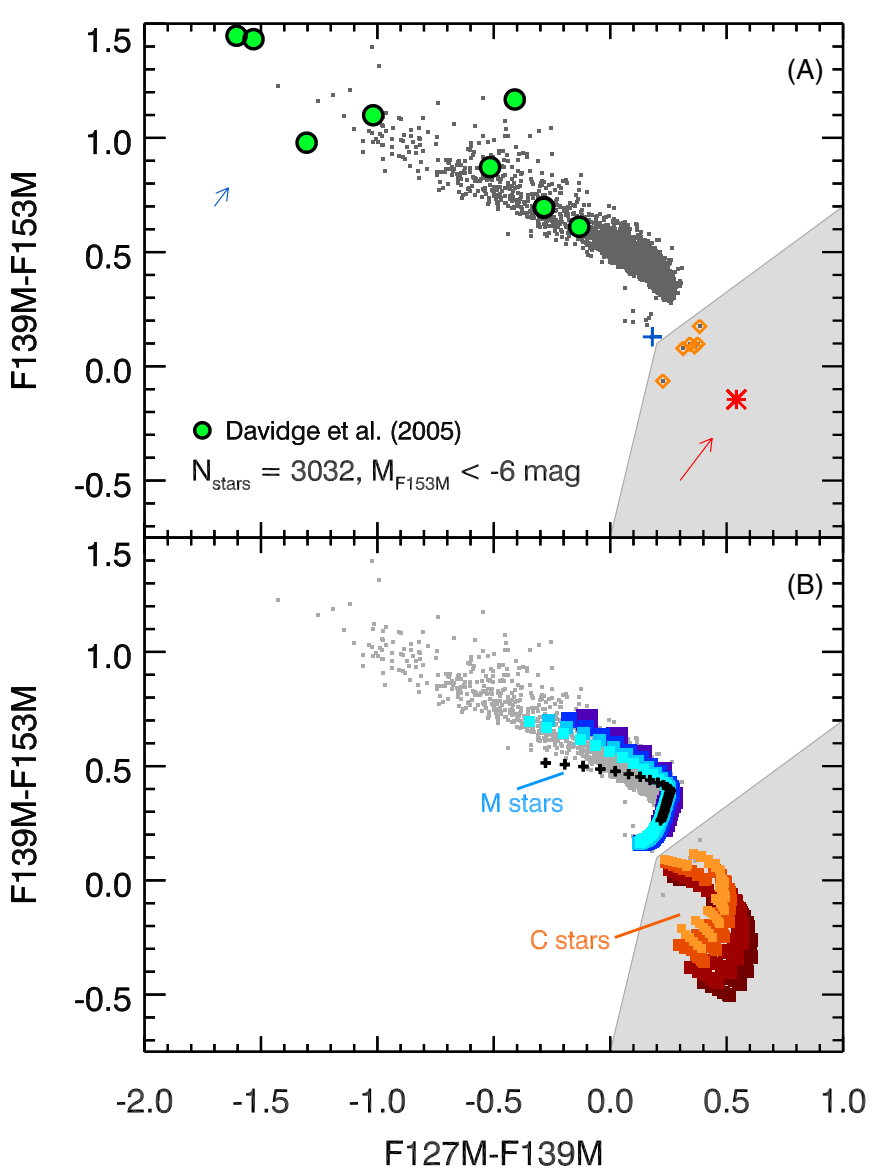

Figure 5. Color-color diagram comparing the WFC3/IR colors to the model predictions for $\mathrm{C}$ and $\mathrm{M}$ giants. Panel (A) shows the observed colors of AGB stars, with red stars from Davidge et al. (2005) plotted as green circles and candidate $\mathrm{C}$ stars marked as in Figure 3. Only stars brighter than the TRGB $\left(M_{\mathrm{F} 153 \mathrm{M}}<-6 \mathrm{mag}\right)$ are included to eliminate most red giant branch stars. Circumstellar reddening vectors for dust-enshrouded stars are plotted with blue $\left(\mathrm{M}\right.$ star) and red $\left(\mathrm{C}\right.$ star) arrows for $E\left(J-K_{\mathrm{S}}\right)=1.0$ mag (corresponding to a mass-loss rate of $\left.\approx 10^{-6} M_{\odot} \mathrm{yr}^{-1}\right)$. These vectors are derived from Groenewegen (2006b) radiative transfer models applied to cool M and C stellar spectra, with $60 \%$ silicate $+40 \%$ AlOx, and $70 \%$ amorphous carbon $+15 \%$ $\mathrm{SiC}$ mixtures, respectively (see Marigo et al. 2008). In panel (B), AGB star models are plotted over the data. The M star models (B. Aringer et al., in preparation) are plotted as blue boxes (using the water line list from Jørgensen et al. 2001), with darker colors representing lower surface gravity, in the range $2 \geqslant \log g\left[\mathrm{~cm} \mathrm{~s}^{-2}\right] \geqslant-0.5$. The $T_{\text {eff }}$ decreases from bottom to top along this sequence, with values in the range $5000 \leqslant T_{\text {eff }} \leqslant 2600 \mathrm{~K}$ : the kink with the reddest $\mathrm{F} 127-\mathrm{F} 139 \mathrm{M}$ colors is at $T_{\mathrm{eff}} \simeq 3300 \mathrm{~K}$. For reference, black pluses show an example of the same $\mathrm{M}$ star models $\left(\log g=0.5\left[\mathrm{~cm} \mathrm{~s}^{-2}\right]\right)$ using a different water line list (Barber et al. 2006). The $\mathrm{C}$ star models (Aringer et al. 2009) are plotted as red boxes, with darker colors representing higher atmospheric $\mathrm{C} / \mathrm{O}$ ratios, over the range $1.05<\mathrm{C} / \mathrm{O}<2$. They span $4000 \mathrm{~K} \leqslant T_{\text {eff }} \leqslant 2400 \mathrm{~K}$ and $0 \geqslant \log g\left[\mathrm{~cm} \mathrm{~s}^{-2}\right] \geqslant-1$, with the coolest $\mathrm{C}$ star models now being located at the bottom of the drawn sequences. All models assume a solar abundance for the heavy metals. The shaded region marks the area where $\mathrm{C}$ stars are expected.

(A color version of this figure is available in the online journal.)

In Figure 5, we show only stars above the TRGB. However, inclusion of stars up to 1 mag fainter than the F153M TRGB reveals no additional $\mathrm{C}$ star candidates in the shaded region. Therefore, according to the radiative transfer models of Groenewegen (2006b) and assuming a dust mixture of $70 \%$ amorphous carbon $+15 \% \mathrm{SiC}, \mathrm{C}$ stars that are undetected here due to circumstellar extinction (those fainter than 1 mag below the F153M TRGB) must have mass-loss rates in excess of $\sim 10^{-5} M_{\odot} \mathrm{yr}^{-1}$. In the Magellanic Clouds, $<1.5 \%$ of $\mathrm{C}$ stars 
have mass-loss rates higher than this (assuming solar gas-to-dust ratio, $\psi_{\odot}=500$; Boyer et al. 2012; Riebel et al. 2012). Thus, with 1-7 detected $\mathrm{C}$ star candidates, we expect $<0.1$ additional dusty $\mathrm{C}$ stars in this field. Including this in the final $\mathrm{C} / \mathrm{M}$ ratio results in $\mathrm{C} / \mathrm{M}=(1-7.1) /(3025-3032)=\left(3.3_{-0.1}^{+20}\right) \times 10^{-4}$, with the lower limit derived from the uncertainty in the number of M stars.

\section{INTERPRETATION/DISCUSSION}

A few searches for carbon stars in M31 using optical photometry with broad and narrow filters have successfully identified AGB stars. Brewer et al. $(1995,1996)$ carried out a photometric narrow-band survey along with a spectroscopic follow up of five fields southwest of the center of M31 along the major axis (Figure 2) and found a total of $243 \mathrm{C}$ stars. More recently, Nowotny et al. (2001), Battinelli et al. (2003), and Battinelli \& Demers (2005) conducted additional searches along the same southwest axis, using similar techniques. The resulting relationship between metallicity and the $\mathrm{C} / \mathrm{M}$ ratio in $\mathrm{M} 31$ is shown in Figure 1.

We can use this relationship to predict the number of $\mathrm{C}$ stars expected in this single field (not including the addition of very dusty, optically obscured $\mathrm{C}$ stars that are missing from Figure 1). We assume that the surveyed field follows the metallicity gradient across the bulge, derived by Saglia et al. (2010), who measure the abundances via optical long-slit data along six position angles, including one that passes very near this field $\left(0.05 \lesssim[\mathrm{M} / \mathrm{H}] \lesssim 0.2\right.$ at $\left.R_{\mathrm{M} 31}=2 \mathrm{kpc}\right)$. A line fit through all data points in Figure 1 thus predicts $40-64 \mathrm{C}$ stars. The range reflects both the $3 \sigma$ uncertainty in the line fit and the range in metallicity. Using only the points from Brewer et al. (1995) predicts $11-40 \mathrm{C}$ stars, and the lowest $\mathrm{C} / \mathrm{M}$ ratio shown in Figure 1 yields $57 \pm 3 \mathrm{C}$ stars. Here, we investigate the discrepancy between these predictions and the observed number of C star candidates (Section 2.1).

\subsection{Are the Missing M31 C Stars Fainter than Our Detection Limit?}

Crowding is smooth across the field of view, at approximately 3.5 stars $\operatorname{arcsec}^{-2}$, so it does not affect the completeness at different positions. Based on false star tests (Section 2), the $50 \%$ completeness limit is $21.6-22.8 \mathrm{mag}$, depending on the filter. Since the TRGB is $>3$ mag brighter than the $50 \%$ completeness limit at all positions, photometric incompleteness has not affected our C star sample. Indeed, the false star tests indicate that the F153M photometry is $99 \%$ complete at the TRGB.

Circumstellar extinction could also cause carbon stars to fall below our detection limit. However, as described in Section 2.1, the extreme reddening required indicates that we have missed $<1 \mathrm{C}$ star due to circumstellar dust.

\subsection{Are We Confident that C Star Models Are Correct?}

Our C-M classification (Figure 5) is heavily based on the behavior of the $\mathrm{C}$ and $\mathrm{M}$ spectral models from Aringer et al. (2009) and B. Aringer et al. (in preparation), which should therefore be verified. The Infrared Telescope Facility (IRTF) database includes NIR spectra of $\approx 200$ Galactic cool giant stars (Rayner et al. 2009). Figure 4 illustrates that all observed features are predicted by the model spectra from Aringer et al. (2009) and B. Aringer et al. (in preparation). However, because of significant telluric absorption from water and methane from
$1.35-1.42 \mu \mathrm{m}(<20 \%$ transmission $)$, spectral features that cause $\mathrm{C}$ and $\mathrm{M}$ stars to separate in Figure 5 are not observable from the ground.

As an alternative, we can turn to space-based NIR spectral observations. Specifically, AGB stars in the LMC cluster NGC 1850 were observed by the HST program 11913 (PI: J. MacKenty) with the WFC3/IR grism G141, thus providing spectra that avoid the problem of telluric absorption. Figure 4(B) shows the spectra extracted for the C star 2MASS J05085008$6845188\left(J-K_{\mathrm{s}}=1.65 \mathrm{mag}\right)$, which reveals no unexpected features in the F139M filter that might account for the apparent lack of $\mathrm{C}$ stars in Figure 5.

Bright M stars in the same LMC dataset present $J-K_{\mathrm{s}} \simeq$ $1.0 \mathrm{mag}$ and generally flat spectra across G141, as exemplified by the spectrum of 2MASS J05084871-6846220 in Figure 4(A). These flat spectra are identified with $\mathrm{M}$-giant models of $T_{\text {eff }} \gtrsim$ $3300 \mathrm{~K}$, or equivalently with early-M subtypes, in which the $1.3-1.55 \mu \mathrm{m}$ water absorption feature is weak.

\subsection{Stars or Late M-giants?}

Our observations show that stars with the reddest $J H K$ colors from Davidge et al. (2005) are likely late-M giants (i.e., very cool AGB stars, but with O-dominant atmospheres) - their colors in Figure 5 point to the presence of deep water absorption features, which depress the F139M flux and cause red F139M - F153M and blue F127M - F139M colors. The M giant sequence is well described by B. Aringer et al. (in preparation) models using the Jørgensen et al. (2001) water line list. However, difficulty in modeling dynamical atmospheres (pulsation and mass loss) truncates the model sequence at $\mathrm{F} 127 \mathrm{M}-\mathrm{F} 139 \mathrm{M} \approx-0.5 \mathrm{mag}$.

These stars were identified as $\mathrm{C}$ star candidates by Davidge et al. (2005) since their red colors $(J-K>1.3 \mathrm{mag})$ are usually markers for C-rich stars in the Magellanic Clouds and other nearby galaxies (e.g., Groenewegen et al. 2009; Nikolaev $\&$ Weinberg 2000). However, our observations clearly indicate that this is not the case in M31. In the metal-poor LMC, the O-rich AGB stars are relatively warm and rarely reach spectral subtypes later than M3 (e.g., Elias et al. 1985; Frogel et al. 1990; Massey \& Olsen 2003). On the other hand, in the metal rich M31, O-rich AGB stars fall along a cooler Hayashi line (cf. Marigo et al. 2013), which ensures a large fraction of them appearing as late-M subtypes. The same occurs in our own Galaxy and its Bulge, which are known to contain M7-M9 giants in copious numbers (e.g., Frogel \& Whitford 1987; Rayner et al. 2009). This fact is well represented in Figure 6, where we see that comparably low effective temperatures of a $\mathrm{C}$-rich model with metallicity $[\mathrm{M} / \mathrm{H}]=-0.3$ (typical of the $\mathrm{LMC}$ ), are reached by O-rich models with high metallicity (solar and super-solar, reasonably suitable for the inner M31 disk).

There are several examples of known late-M giant stars that show very red $J-K$ colors (e.g., Marshall et al. 2004; van Loon et al. 2005; Javadi et al. 2013), including WOH G64, an OH/IR star with $T_{\text {eff }} \approx 3000 \mathrm{~K}$. However, these red $\mathrm{M}$ giants are vastly outnumbered by red $\mathrm{C}$ stars in galaxies such as the Magellanic Clouds and M33, where most O-rich AGB stars are warmer and thus have early M-type spectral classifications (or even late-K). Inspection of optical spectra from Olsen et al. (2011) of AGB stars in the Magellanic Clouds indicate that only $\approx 3 \%$ of $\mathrm{M}$ stars show $H-K>0.4 \mathrm{mag}$. The $H S T$ data indicate that this fraction increases to $\approx 30 \%$ in this region of M31 (presumably owing to the higher metallicity), assuming that stars that show WFC3/IR colors similar to the Davidge et al. (2005) stars will also show similar $J H K$ colors. 


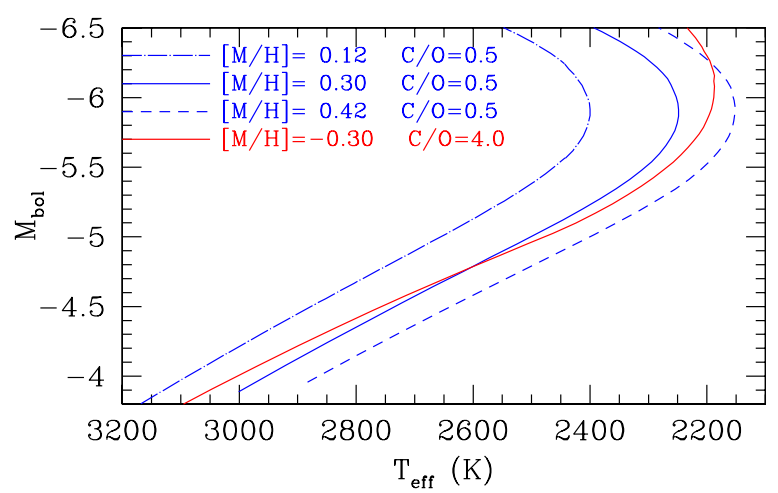

Figure 6. TP-AGB Hayashi lines for a model with $M=1.4 M_{\odot}$ at varying initial metallicity $([\mathrm{M} / \mathrm{H}])$ and surface $\mathrm{C} / \mathrm{O}$ ratio, as indicated. At a given bolometric magnitude, the effective temperature is obtained from integrations of complete atmosphere and envelope models (Marigo et al. 2013) that include on-the-fly computation of both molecular chemistry and opacity (Marigo \& Aringer 2009). (A color version of this figure is available in the online journal.)

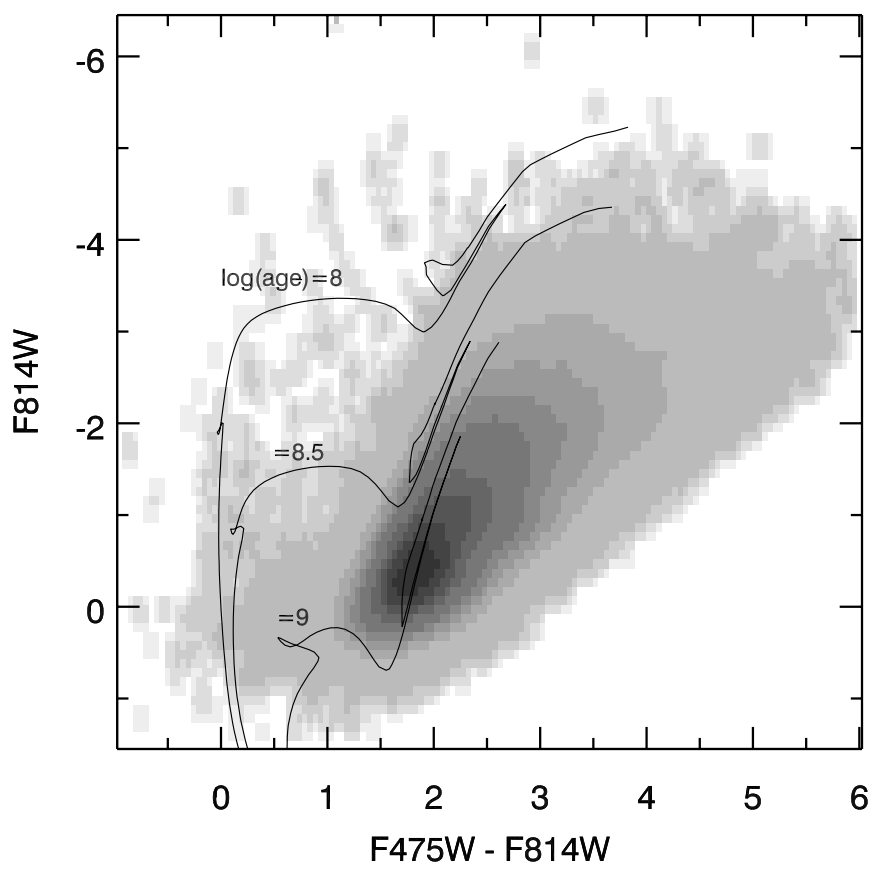

Figure 7. Optical CMD of the observed region, from the PHAT program (Dalcanton et al. 2012a). Padova isochrones are plotted with $A(V)=0.3 \mathrm{mag}$ for $\log ($ age $)=8,8.5$, and 9 (PARSEC, version 1.1; Marigo et al. 2008; Girardi et al. 2010). The CMD indicates the presence of main sequence turn-off stars at least as young as $\sim 300 \mathrm{Myr}$ (or $M \lesssim 4 M_{\odot}$ ).

\subsection{Where Are the C Stars in This Region of M31?}

Age effect? In old, metal-rich stellar populations like the M31 bulge, carbon stars are not expected since the efficiency of the 3DU $(\lambda)$ is not sufficient for stars with $M \lesssim 2 M_{\odot}$ (Karakas et al. 2002). However, from Dorman et al. (2012), we estimate the bulge contributes only $28 \%$ of the $I$-band luminosity in this region. The $I$-band shows minimal contamination from short-lived bright sources (Melbourne \& Boyer 2013), so this fraction indicates that the bulk of the stellar mass in our observed field originates from the younger stellar population of the disk. Models from Courteau et al. (2011) fit to the $3.6 \mu \mathrm{m}$ luminosity profile also indicate that the disk population dominates at $2 \mathrm{kpc}$.

PHAT optical CMDs for this region (Figure 7) also reveal the presence of main sequence turn-off stars younger than $\sim 1 \mathrm{Gyr}$, indicating the presence of $\mathrm{AGB}$ stars with masses $\approx 3-5 M_{\odot}$.

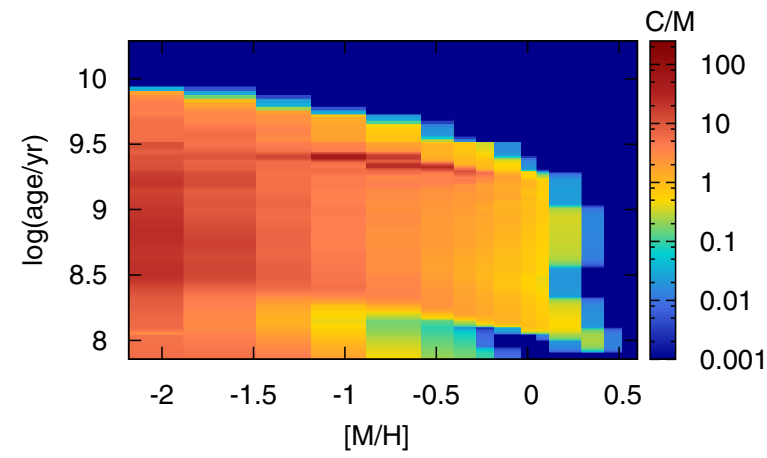

Figure 8. Map of the $\mathrm{C} / \mathrm{M}$ ratio in the age-metallicity plane, according to the new TP-AGB models computed by Marigo et al. (2013). The lack of the C star phase $(C / M=0)$ corresponds to the darkest blue region, which extends toward younger ages at increasing metallicity. Above some critical value of $[\mathrm{M} / \mathrm{H}]$, models predict $\mathrm{C} / \mathrm{M}=0$ over the entire age range. According to Saglia et al. (2010), the metallicity of the region observed here is $[\mathrm{M} / \mathrm{H}] \approx 0.1 \pm 0.1$.

(A color version of this figure is available in the online journal.)

With a stellar population of this age, we expect a much larger population of $\mathrm{C}$ stars based on the $\mathrm{C} / \mathrm{M}$ relationship implied in Figure 1.

Metallicity effect? Brewer et al. (1996) indicate that the $\mathrm{C} / \mathrm{M}$ ratio rapidly decreases toward the inner M31 disk (down to values of $\sim 0.019$ at $R_{\mathrm{M} 31}=4 \mathrm{kpc}$ ) and interpret this decrease as being driven by the metallicity gradient in M31. Our data at $\sim 2 \mathrm{kpc}$ puts additional constraints in the way the $\mathrm{C} / \mathrm{M}$ ratio decreases toward the M31 center. Within $2 \mathrm{kpc}$ of M31's center, Saglia et al. (2010) find that the metallicity spans $0 \lesssim[\mathrm{M} / \mathrm{H}] \lesssim 0.5$, with $[\mathrm{M} / \mathrm{H}] \approx 0.1 \pm 0.1$ near the position of our observations (see their Figure 12). If our very low $\mathrm{C} / \mathrm{M}$ ratio is caused by the high metallicity at this radius, it supports the presence of a metallicity threshold above which C stars simply do not form.

A reduction in the $\mathrm{C} / \mathrm{M}$ ratio with metallicity is indeed indicated by all modern TP-AGB models (Karakas et al. 2002; Marigo et al. 2013) as a result of two effects: the larger amount of carbon that needs to be dredged-up to make the $\mathrm{C} / \mathrm{O}>$ 1 transition, and the fact that the 3DU starts later (at higher luminosities) and is less efficient at increasing metallicity. Figure 8 shows the $\mathrm{C} / \mathrm{M}$ ratio according to the new TP-AGB models from Marigo et al. (2013). For each combination of age (or initial mass) and initial metallicity, the quantity $\mathrm{C} / \mathrm{M}$ is obtained as $\tau_{C} / \tau_{M}$, i.e., the ratio of the lifetime spent by the star in the $\mathrm{C}$-rich mode $(\mathrm{C} / \mathrm{O}>1)$ over the one spent in the O-rich mode $(\mathrm{C} / \mathrm{O}<1)$. To avoid contamination from the RGB phase, both $\tau_{\mathrm{C}}$ and $\tau_{\mathrm{M}}$ are evaluated for bolometric magnitudes $M_{\text {bol }}<-3.6 \mathrm{mag}$. The region of $\mathrm{C} / \mathrm{M}=0$ (dark blue in Figure 8 ) extends to younger ages at increasing metallicity, encompassing the whole age range for $[\mathrm{M} / \mathrm{H}] \gtrsim 0.4$. At these metallicities, more massive TP-AGB stars (ages $\lesssim 1$ Gyr) go through a few dredge-up events, but the termination of the TP-AGB phase (determined by the onset of the super-wind mass loss) occurs before $\mathrm{C} / \mathrm{O}$ can exceed unity. The models from Marigo et al. (2013) also indicate that, while the trend depicted in Figure 8 has a general validity, the exact upper limit in metallicity for the formation of $\mathrm{C}$ stars is sensitive to model details, mainly related to the 3DU and mass loss. In this context, observations like the ones we present in this work are of paramount importance to set stringent constraints on the evolution of TP-AGB stars in the still largely unexplored regime of high metallicity. 


\section{CONCLUSIONS}

We have conducted a search for carbon-rich AGB stars in the inner disk of M31 using the WFC3/IR medium-band filters on board HST. We find that:

1. WFC3/IR medium filters are very efficient in classifying AGB stars into their O- and C-rich subtypes, which is important for extending $\mathrm{C}$ star searches in galaxies outside of the Local Group where resolution and sensitivity prevent ground-based NIR imaging.

2. These filters also place O-rich $\mathrm{M}$ stars along a sequence of increasing water absorption, with the potential of providing information about the $T_{\text {eff }}$ distribution of these stars. Given the proximity between the F127M and F153M filters, this classification is largely unaffected by star-to-star variations in the reddening.

3. Contrary to previous work, our observations reveal the near absence of $\mathrm{C}$ stars in this inner disk region of $\mathrm{M} 31$. With a sample of 3032 TP-AGB stars, we find $(\mathrm{C} / \mathrm{M})=$ $\left(3.3_{-0.1}^{+20}\right) \times 10^{-4}$, one to two orders of magnitude lower than previous C star searches in M31.

4. This study is among the first to search for complete, unbiased populations of $\mathrm{C}$ stars at $[\mathrm{M} / \mathrm{H}] \gtrsim 0$, and is expected to provide stringent constraints to evolutionary models of metal-rich AGB stars. The extremely low observed C/M ratio suggests a metallicity ceiling for $\mathrm{C}$ stars to form. Our results put this ceiling at $[\mathrm{M} / \mathrm{H}] \approx 0.1 \mathrm{dex}$, which is the estimated mean metallicity of this M31 region.

5. More observations in these filters across M31 and other galaxies are strongly needed both for studies of AGB stellar evolution, dust production, and mass loss.

We thank the referee, Jacco van Loon, for thoughtful comments that improved the manuscript and helped to clarify important issues. This work was supported by the NASA Postdoctoral Program at the Goddard Space Flight Center, administered by ORAU through a contract with NASA and by NASA through HST grant numbers GO-12862 and GO-12055 from the STScI, which is operated by AURA, Inc., under NASA contract NAS5-26555. P.M. and L.G. acknowledge support from Progetto di Ateneo 2012, University of Padova, ID: CPDA125588/12. This research was funded in part by the Austrian Science Fund (FWF): P21988-N16.

Facility: HST (WFC3)

\section{REFERENCES}

Aringer, B., Girardi, L., Nowotny, W., Marigo, P., \& Lederer, M. T. 2009, A\&A, 503, 913

Azzopardi, M., Rebeirot, E., Lequeux, J., \& Westerlund, B. E. 1991, A\&AS, 88,265

Barber, R. J., Tennyson, J., Harris, G. J., \& Tolchenov, R. N. 2006, MNRAS, 368, 1087

Barmby, P., Ashby, M. L. N., Bianchi, L., et al. 2006, ApJL, 650, L45

Barmby, P., Huchra, J. P., Brodie, J. P., et al. 2000, AJ, 119, 727

Battinelli, P., \& Demers, S. 2005, A\&A, 430, 905

Battinelli, P., \& Demers, S. 2009, A\&A, 493, 1075

Battinelli, P., Demers, S., \& Letarte, B. 2003, AJ, 125, 1298

Battinelli, P., Demers, S., \& Mannucci, F. 2007, A\&A, 474, 35

Boyer, M. L., Skillman, E. D., van Loon, J. Th., Gehrz, R. D., \& Woodward, C. E. 2009, ApJ, 697, 1993

Boyer, M. L., Srinivasan, S., Riebel, D., et al. 2012, ApJ, 748, 40

Boyer, M. L., Srinivasan, S., van Loon, J. Th., et al. 2011, AJ, 142, 103
Brewer, J. P., Richer, H. B., \& Crabtree, D. R. 1995, AJ, 109, 2480

Brewer, J. P., Richer, H. B., \& Crabtree, D. R. 1996, AJ, 112, 491

Cioni, M.-R. L., Girardi, L., Marigo, P., \& Habing, H. J. 2006, A\&A, 448, 77

Conroy, C., Gunn, J. E., \& White, M. 2009, ApJ, 699, 486

Courteau, S., Widrow, L. M., McDonald, M., et al. 2011, ApJ, 739, 20

Dalcanton, J. J., Williams, B. F., Lang, D., et al. 2012a, ApJS, 200, 18

Dalcanton, J. J., Williams, B. F., Melbourne, J. L., et al. 2012b, ApJS, 198, 6

Davidge, T. J., Olsen, K. A. G., Blum, R., Stephens, A. W., \& Rigaut, F. 2005, AJ, 129,201

Dolphin, A. E. 2000, PASP, 112, 1383

Dorman, C. E., Guhathakurta, P., Fardal, M. A., et al. 2012, ApJ, 752, 147

Elias, J. H., Frogel, J. A., \& Humphreys, R. M. 1985, ApJS, 57, 91

Feast, M. 2007, in ASP Conf. Ser. 378, Why Galaxies Care About AGB Stars: Their Importance as Actors and Probes, ed. F. Kerschbaum, C. Charbonnel, \& R. F. Wing (San Francisco, CA: ASP), 479

Ferrarotti, A. S., \& Gail, H.-P. 2006, A\&A, 447, 553

Frogel, J. A., Mould, J., \& Blanco, V. M. 1990, ApJ, 352, 96

Frogel, J. A., \& Whitford, A. E. 1987, ApJ, 320, 199

Girardi, L., et al. 2010, ApJ, 724, 1030

Groenewegen, M. A. T. 2006a, in Planetary Nebulae Beyond the Milky Way, ed. L. Stanghellini, J. R. Walsh, \& N. G. Douglas (Berlin: Springer), 108

Groenewegen, M. A. T. 2006b, A\&A, 448, 181

Groenewegen, M. A. T. 2007, in ASP Conf. Ser. 378, Why Galaxies Care About AGB Stars: Their Importance as Actors and Probes, ed. F. Kerschbaum, C. Charbonnel, \& R. F. Wing (San Francisco, CA: ASP), 433

Groenewegen, M. A. T., Sloan, G. C., Soszyński, I., \& Petersen, E. A 2009, A\&A, 506, 1277

Hook, R., Stoehr, F., \& Krist, J. 2008, STECF, 44, 11

Javadi, A., van Loon, J. Th., Khosroshahi, H., \& Mirtorabi, M. T. 2013, MNRAS, 432,2824

Jørgensen, U. G., Jensen, P., Sørensen, G. O., \& Aringer, B. 2001, A\&A, 372,249

Karakas, A. I., \& Lattanzio, J. C. 2007, PASA, 24, 103

Karakas, A. I., Lattanzio, J. C., \& Pols, O. R. 2002, PASA, 19, 515

Krist, J. 1995, in ASP Conf. Ser. 77, Astronomical Data Analysis Software and Systems IV, ed. R. A. Shaw, H. E. Payne, \& J. J. E. Hayes (San Francisco, CA: ASP), 349

Maraston, C., Daddi, E., Renzini, A., et al. 2006, ApJ, 652, 85

Marigo, P. 2001, A\&A, 370, 194

Marigo, P., \& Aringer, B. 2009, A\&A, 508, 1539

Marigo, P., Bressan, A., Nanni, A., Girardi, L., \& Pumo, M. L. 2013, MNRAS, in press (arXiv:1305.4485)

Marigo, P., Girardi, L., Bressan, A., et al. 2008, A\&A, 482, 883

Marigo, P., Girardi, L., Bressan, A., et al. 2010, in IAU Symp. 262, Stellar Populations-Planning for the Next Decade, ed. G. R. Bruzual \& S. Charlot (Cambridge: Cambridge Univ. Press), 36

Marshall, J. R., van Loon, J. Th., Matsuura, M., et al. 2004, MNRAS, 355,1348

Massey, P., \& Olsen, K. A. G. 2003, AJ, 126, 2867

Melbourne, J., \& Boyer, M. L. 2013, ApJ, 764, 30

Melbourne, J., Williams, B. F., Dalcanton, J. J., et al. 2012, ApJ, 748, 47

Miszalski, B., Mikołajewska, J., \& Udalski, A. 2013, MNRAS, 432, 3186

Nikolaev, S., \& Weinberg, M. D. 2000, ApJ, 542, 804

Nowotny, W., Aringer, B., Höfner, S., \& Eriksson, K. 2013, A\&A, 552, A20

Nowotny, W., Kerschbaum, F., Schwarz, H. E., \& Olofsson, H. 2001, A\&A, 367,557

Olsen, K. A. G., Blum, R. D., Stephens, A. W., et al. 2006, AJ, 132, 271

Olsen, K. A. G., Zaritsky, D., Blum, R. D., Boyer, M. L., \& Gordon, K. D. 2011, ApJ, 737, 29

Rayner, J. T., Cushing, M. C., \& Vacca, W. D. 2009, ApJS, 185, 289

Riebel, D., Srinivasan, S., Sargent, B., \& Meixner, M. 2012, ApJ, 753, 71

Rieke, G. H., \& Lebofsky, M. J. 1985, ApJ, 288, 618

Rowe, J. F., Richer, H. B., Brewer, J. P., \& Crabtree, D. R. 2005, AJ, 129, 729

Saglia, R. P., Fabricius, M., Bender, R., et al. 2010, A\&A, 509, A61

Sibbons, L. F., Ryan, S. G., Cioni, M.-R. L., Irwin, M., \& Napiwotzki, R. 2012, A\&A, 540, A135

Stephens, A. W., Frogel, J. A., DePoy, D. L., et al. 2003, AJ, 125, 2473

van Loon, J. Th., Cioni, M.-R. L., Zijlstra, A. A., \& Loup, C. 2005, A\&A, 438, 273

Ventura, P., D’ Antona, F., Mazzitelli, I., \& Gratton, R. 2001, ApJL, 550, L65

Whitelock, P. 1993, in IAU Symp. 153, Galactic Bulges, ed. H. Dejonghe \& H. J. Habing (Dordrecht: Kluwer), 39

Zurita, A., \& Bresolin, F. 2012, MNRAS, 427, 1463 